\title{
USO DE LA TÉCNICA DE MAGNETRON SPUTTERING EN EL RECUBRIMIENTO DE MATERIALES PARA APLICACIONES TECNOLÓGICAS
}

\section{USE OF THE MAGNETRON SPUTTERING TECHNIQUE ON THE COATING OF MATERIALS FOR TECHNOLOGICAL APPLICATIONS}

\author{
Jose Antonio Tiburcio Moreno ${ }^{7}$, \\ Hernán Hernández Llamas ${ }^{8}$, \\ Jose Martin Yáñez Limón ${ }^{9}$
}

\section{RESUMEN}

En este artículo se presentan resultados experimentales de recubrimientos de materiales que en la actualidad son atractivos para diferentes aplicaciones industriales, en este trabajo se presenta los primeros resultados de recubrimientos multicapas del sistema CrAI/CrAIN, los cuales fueron depositados en sustratos de vidrio a partir de blancos de Cromo y Aluminio de alta pureza, usando la técnica de magnetrón sputtering. Las monocapas y las multicapas fueron estudiadas individualmente con la finalidad de obtener fases cristalinas estables a través de parámetros de depósito óptimos. La morfología y composición de las fases sintetizadas fueron analizadas mediante SEM (Microscopia Electrónica de Barrido) y EDS (Espectroscopia de Electrones Secundarios), obteniéndose resultados previstos para la estructura cristalina de las películas. Este estudio de acuerdo a los resultados preliminares obtenidos constituye un indicador muy prometedor para potenciales aplicaciones industriales, especialmente en la industria de herramientas de corte.

Palabras clave: recubrimientos, películas delgadas, erosión catódica

\section{ABSTRACT}

In this paper, experimental results of coatings of materials that are currently attractive for different industrial applications are presented. Consequently, a systematic study of multilayer coatings of the CrAl/ CrAIN system was carried out. The coatings were deposited on glass substrates from chromium and aluminum high purity targets, using the magnetron sputtering technique. The monolayers and the multilayers were studied individually in order to obtain stable crystalline phases through optimal deposit parameters. The morphology and composition of the synthesized phases were analyzed by SEM and EDS, this study according to the results obtained is a promising indicator for potential industrial applications, especially in the industry of cutting tools.

Keywords: coatings, thin films, magnetron sputtering

\footnotetext{
7 Universidad Nacional Jorge Basadre Grohmann, Facultad de Ciencias, Av. Miraflores s/n, Ciudad Universitaria, Tacna, Perú.

${ }^{8}$ Universidad Autónoma de Querétaro, Cerro de las Campanas, Centro Universitario, C.P. 76010, Santiago de Querétaro, Querétaro, México

${ }^{9}$ Centro de Investigación y Estudios Avanzados del IPN, Unidad - Querétaro, Libramiento Norponiente N $^{\circ} 2000$, Fraccionamiento Real de Juriquilla, C.P. 76230, Querétaro, México
} 


\section{INTRODUCCIÓN}

Los recubrimientos duros han sido utilizados con éxito desde hace algunos años para proteger materiales e incrementar la vida de herramientas industriales debido a sus excelentes propiedades mecánicas (Gonzáles Hernández \& Espinoza Beltran, 2003). Los materiales más utilizados para este tipo de recubrimiento pertenecen al grupo de los carburos, nitruros y boruros de algunos metales de transición. Estos materiales, aplicados en forma de película delgada aumentan sustancialmente la dureza, reducción al desgaste, resistencia a la corrosión, estabilidad química y poseen un bajo coeficiente de fricción (Ipaz, Aperador, Caicedo, Esteve, \& Zambrano, 2012). Los recubrimientos generalmente se depositan sobre sustratos mediante películas delgadas y se pueden presentar como sistemas monocapas y multicapas. La tecnología para la deposición de estas películas ha experimentado avances importantes, generando procesos en los cuales se puede conseguir películas de muy alta calidad. Las tecnologías de deposición están separadas en procesos físicos y químicos. Sin embargo, existen otros procesos que no pueden ser considerados puramente de un solo tipo por lo cual son clasificados como físico-químicos, tal es el caso de la erosión catódica, procesos de plasma y procesos térmicos. En este trabajo se usa la técnica de la erosión catódica para depositar películas delgadas sobre sustratos de vidrio.

Para recubrimientos tipo monocapa, las propiedades mecánicas y tribológicas pueden atribuirse a la cantidad de material que se usa, las cuales pueden cambiar cuando el volumen del material disminuye y aumenta la relación superficie/volumen. La razón de estos cambios se debe principalmente a la microestructura y morfología de las capas. Asi como también, en la naturaleza de sus enlaces que se forman durante el depósito de las películas sobre el sustrato (Gonzales, Cadenas, Fernandez, Cortizo, \& Rodriguez, 2007). Las condiciones de crecimiento de una película generalmente impiden que se alcance un equilibrio en los átomos que llegan a la superficie del sustrato durante la etapa de formación. Esto provoca que la composición y estructura sea diferente a la que se llegaría por medio de la síntesis del material en un volumen mayor. Así mismo, estos cambios en la estructura pueden crear un alto número de defectos en la capa. Estos defectos crean fenómenos asociados a la morfología de las capas. Se puede establecer entonces que una monocapa delgada puede ser considerada como tal cuando, en un espesor determinado, los fenómenos de superficie se presentan más que aquellos fenómenos presentes en el material en sí (Holmberg \& Mathews, 1994).

En la técnica de erosión catódica o "Sputtering" los átomos de la superficie de un blanco sólido a través del impacto de un gas ionizado generado por la presencia de un plasma son separados de la superficie, en este trabajo de investigación fueron gases inertes como $\mathrm{Ar}^{+}{\text {y } \mathrm{Kr}^{+}}^{+}$(S. Xu, Long, Sim, Hoong, \& Ostrikov, 2005). Este método está relacionado a la transferencia de momento de partículas energéticas a los átomos de la superficie. Otros factores a tomar en cuenta en un proceso de erosión catódica son la presión de trabajo, el tipo de gas, el tipo de blanco y la densidad de corriente, pues estos parámetros influyen en la velocidad de depósito. Los procesos de Sputtering se dividen, en general, en 4 categorías: DC (corriente continua), RF (radio frecuencia), Magnetrón y Reactivo (Shigesato \& Paine, 1994). En el desarrollo de la presente investigación se usa el método de magnetrón sputtering DC.

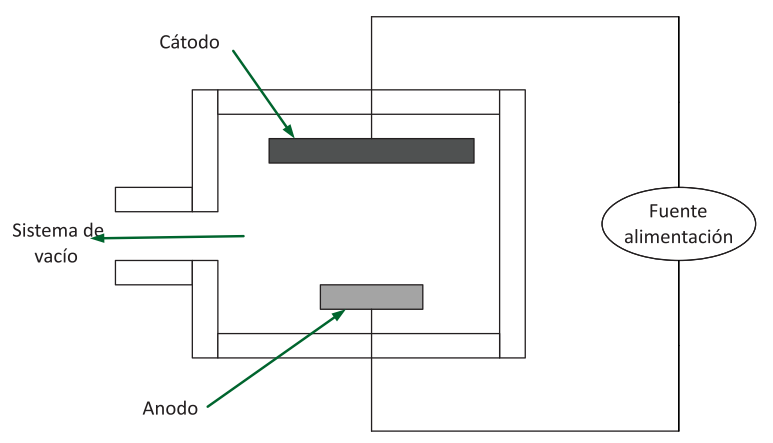

Figura 1: esquema simplificado de un sistema de sputtering. 
En este sistema, el plasma se inicia entre el ánodo (sustrato) y el cátodo (blanco) en un ambiente de baja presión y por la aplicación de un voltaje alto DC. El plasma se mantiene por la aceleración de los electrones secundarios emitidos a partir del cátodo a causa del bombardeo iónico. En general se usa $\mathrm{Ar}$ como gas de trabajo por su tamaño atómico (X. Xu, Wu, Zhang, \& Jin, 2001). El cátodo con magnetrón se diferencia del cátodo convencional por la presencia del campo magnético. La polaridad del campo magnético ayuda a la orientación de los átomos.

Los blancos pueden ser de materiales individuales puros, aleaciones, o de tipo mosaico. Los blancos tipo mosaico se refieren a aquellos que son utilizados si se desea depositar dos o más materiales al mismo tiempo.

Uno de los componentes de nuestras películas es el cromo que es usado principalmente como recubrimiento protector a la corrosión, al calor y como recubrimiento para galvanizados. El cromo es capaz de formar compuestos con otros elementos en estados de oxidación. Este elemento reacciona con el nitrógeno para formar nitruro de cromo (III), que es un compuesto inorgánico formado por un átomo de cromo y un átomo de nitrógeno $(\mathrm{CrN})$. Los nitruros basados en cromo poseen una relativa alta dureza y alta resistencia al desgaste (Velasco et al., 2001). El nitruro de cromo generalmente puede ser obtenido mediante la combinación directa de cromo con nitrógeno en temperaturas mayores a $800^{\circ} \mathrm{C}$ o por técnicas de deposición física de vapor como lo es la erosión catódica. Su estructura cristalina es cubica simple. También, con el propósito de mejorar las propiedades mecánicas de los recubrimientos se añade aluminio que es un elemento químico utilizado para formar aleaciones con otros elementos para aumentar su resistencia y adquirir nuevas propiedades. Las aleaciones de aluminio son ligeras, fuertes y de fácil formación (Bin, Xiaodong, Naisai, \& Jiawen, 2000) . El aluminio también es muy resistente a la corrosión cuando se encuentra altamente puro. Como ya mencionamos, los nitruros de elementos metálicos de transición han sido ampliamente utilizados como recubrimientos duros, para proteger del desgaste y la corrosión, en herramientas de corte y modelamiento debido a sus excelentes propiedades mecánicas y tribológicas. Entre estos recubrimientos, el CrAIN es, probablemente, el material más prometedor como película protectora debido a su excelente resistencia a la oxidación, estabilidad térmica, alta dureza y buenas propiedades tribológicas en comparación a otros materiales (por ejemplo, TiAIN) (Nouveau, Tlili, Aknouche, Benlatreche, \& Patel, 2012)

Estas propiedades se presentan en este material cuando presenta una estructura cubica simple en donde el Al sustituye al metal de la red, en este caso al $\mathrm{Cr}$, en la red del CrN. Esto nos da una relación en la red de $\mathrm{Cr}_{1-x} \mathrm{Al}_{x} \mathrm{~N}$ (Li, Chen, Wang, Yang, \& Wu, 2013). El efecto de la concentración de Al en el sistema CrAIN es notado en las propiedades mecánicas (estabilidad termal y resistencia a la oxidación) y, en altas cantidades de Al, en el aumento de dureza y porosidad del material (Beliardouh, Bouzid, Nouveau, Tlili, \& Walock, 2015).

El objetivo central de este trabajo fue caracterizar y analizar la microestructura y morfología de recubrimientos de CrAIN y CrAl/CrAIN depositados en la superficie de sustratos de vidrio, para considerar potenciales aplicaciones en la industria metalmecánica y herramientas de corte.

\section{Materiales, procedimiento y equipos}

Reactivos químicos

Los reactivos químicos etanol, xileno y acetona usados para la limpieza de los sustratos de vidrio fueron adquiridos de Sigma-Aldrich todos en grado de alta pureza.

Preparación de los sustratos

El depósito de los recubrimientos se realizó en sustratos de vidrio, los cuales fueron preparados para mantener su limpieza durante el proceso de depósito mediante una solución de etanol, xileno y acetona agitada en un equipo de limpieza ultrasónica 
durante 8 minutos. Luego, los sustratos se depositaron en un recipiente que solo contiene etanol y colocado nuevamente al equipo de ultrasonido por un periodo de 8 minutos, tal como se muestra en la Figura 1. Posterior a este tratamiento los sustratos fueron retirados del recipiente para ser secados con aire comprimido para eliminar todo residuo de humedad y almacenados en bolsas individuales con su respetiva etiqueta.
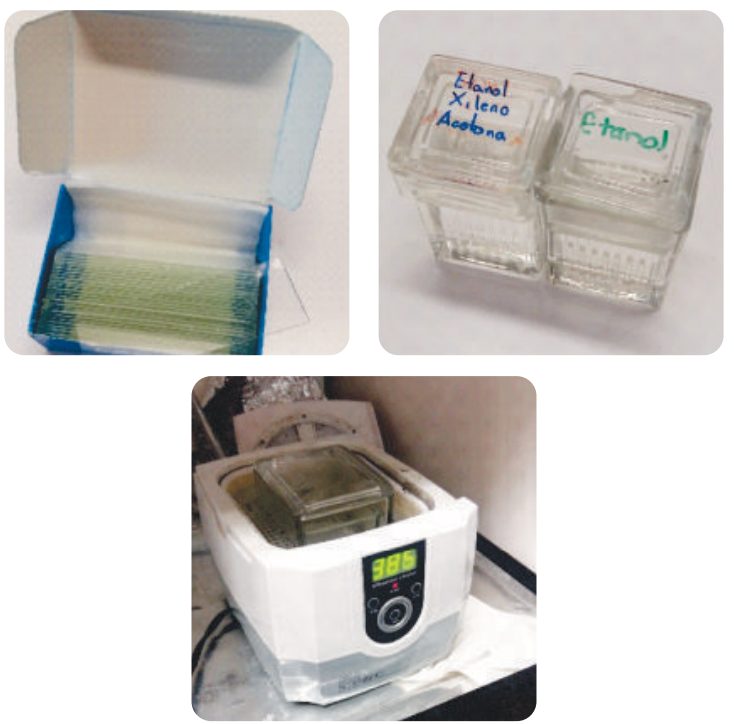

Figura 2: Componentes para limpieza de sustratos, a) sustratos de vidrio, b) reactivos químicos y c) equipo de limpieza ultrasónico.

\section{Depósitos de los recubrimientos}

Se depositaron los recubrimientos en monocapas de CrAl y CrAIN y multicapas (CrAl/CrAIN y CrAIN/CrAl), considerando los parámetros de trabajo: flujo de Nitrógeno, tiempo de depósito, potencia y presión de trabajo. El proceso de depósito fue realizado en un equipo de sputtering Intercovamex $V 3$, usando $\operatorname{Ar}$ y $\mathrm{N}_{2}$ como gases de trabajo y se usó un blanco tipo mosaico, tal como se muestra en la Figura 2, de aproximadamente 2 pulgadas de diámetro, el equipo cuenta con una bomba mecánica y una turbina para producir vacío en rangos de hasta $10^{-5} \mathrm{mbar}$.

\section{Caracterización de las películas depositadas}

Para determinar el espesor y rugosidad de las películas se utilizó un perfilometro marca
Bruker modelo Contour G InMotion 3D, la morfología de las muestras se realizó mediante un microscopio electrónico de barrido JEOL JSM-7610F FEG-SEM y el análisis de la composición química se realizó con un equipo de espectroscopia dispersiva de Rayos X Phillip XL-30 ESEM.
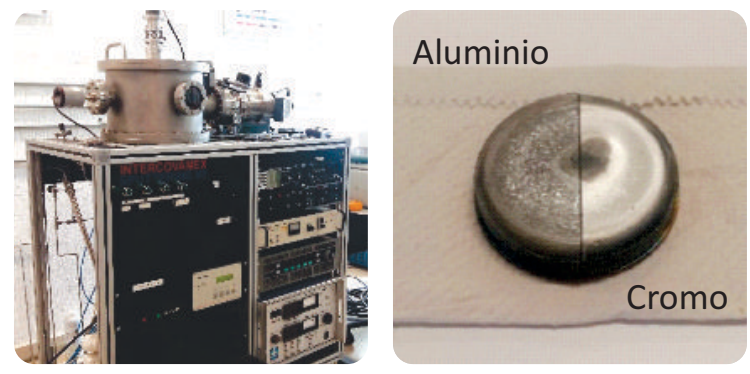

Figura 3: a) Equipo de erosión catódica y b) blanco tipo mosaico binario de cromo y aluminio

Los sustratos de vidrio se fijaron en el porta muestras, se muestra en la Figura 3 , en posiciones adecuadas y luego colocados en la cámara del equipo sputtering, para proceder a realizar el vacío en la misma mediante una bomba mecánica y turbina. Para el control de la presión se usó un sensor de cátodo frio.

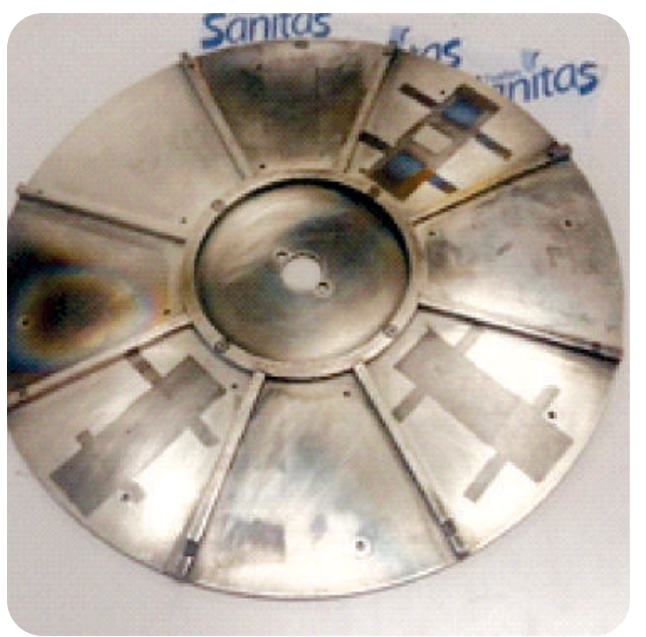




\section{Resultados y discusiones}

Los parámetros de trabajo utilizados en los depósitos fueron los que se indican en la Tabla 1.

Tabla 1. Parámetros utilizados

\begin{tabular}{|c|c|}
\hline Temperatura de trabajo & $27^{\circ} \mathrm{C}$ \\
\hline Presión de Trabajo & $2.0-3.5 \times 10^{-2} \mathrm{mbar}$ \\
\hline Distancia al blanco & $8 \mathrm{~cm}$ \\
\hline Potencia & $30 / 60 \mathrm{~W}$ \\
\hline Relación Ar/N & $80 / 20,40 / 60,0 / 100$ \\
\hline Blanco & Mosaico Cr/Al \\
\hline
\end{tabular}

La fuente de tensión del equipo sputtering tiene varios modos de trabajo que permiten mantener un control estable de la potencia, la corriente o el voltaje, según el modo en el que se encuentren. Usualmente se trabaja con el modo potencia pues es la variable que interesa controlar. Sin embargo, para mantener estable el funcionamiento de la fuente fue necesario cambiar al modo voltaje lo cual fue útil para para controlar la potencia hasta $30 \mathrm{~W}$ para depósitos con el gas de trabajo Ar y $60 \mathrm{~W}$ cuando el gas de trabajo fue Nitrógeno o en la combinación de $\mathrm{Ar} / \mathrm{N}$, para mantener la potencia estable los depósitos se realizaron en periodos de 15 minutos intermitentes hasta completar el tiempo del depósito de 1 hora.

Como se puede apreciar en la figura 5, las películas depositadas con los parámetros mencionados anteriormente.

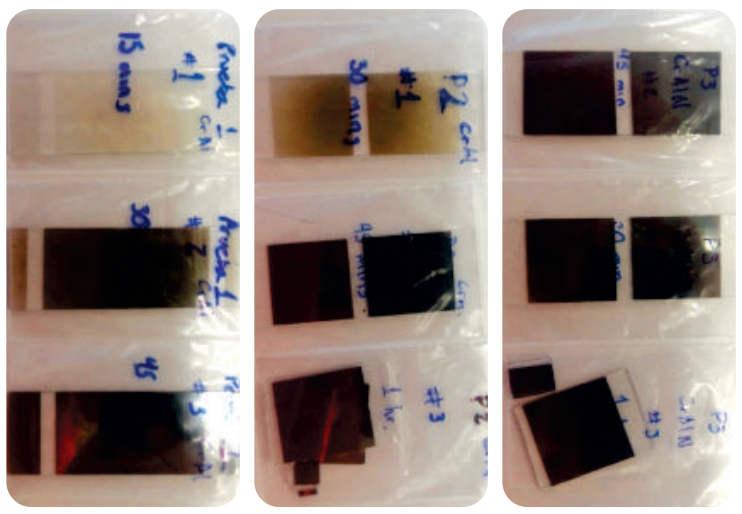

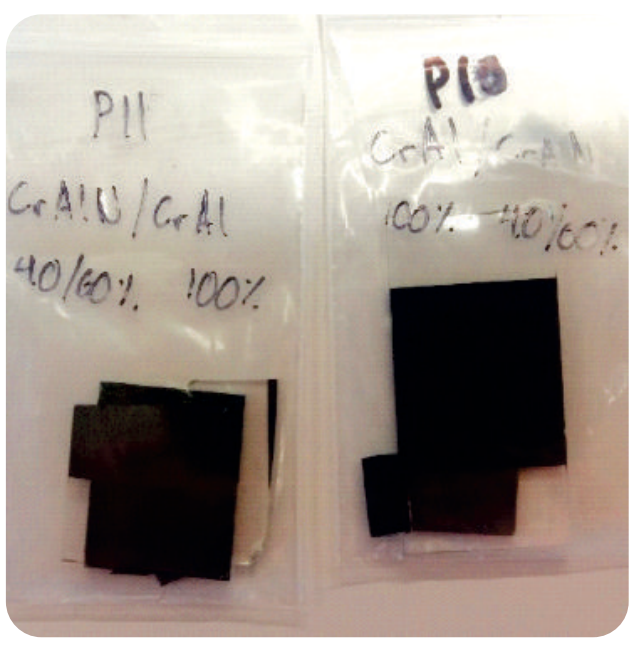

Figura 5. Sustratos de vidrio con depósitos de bicapas CrAIN/CrAl

La medida de la rugosidad de la superficie $\left(R_{a}\right)$ y el espesor $\left(z_{a}\right)$ de las películas depositadas se realizó en un equipo de perfilometría marca Bruker modelo ContourGTInMotion 3D. Las imágenes obtenidas por este equipo recibieron un tratamiento posterior con el software de acceso libre Gwyddion. Los resultados de estas mediciones se pueden observar en la tabla 2.

Tabla 2. Resultados de perfilometría

\begin{tabular}{|c|c|c|}
\hline Película & $\mathrm{R}_{\mathrm{a}}(\mu \mathrm{m})$ & $\mathrm{z}_{\mathrm{a}}(\mu \mathrm{m})$ \\
\hline P3 CrALN 60 min & 0.249 & 1,0523 \\
\hline P6 CrAIN $100 \%$ N & 0.086 & 0.3054 \\
\hline $\begin{array}{c}\text { P10 CrAl/CrAIN } \\
40 / 60 \% \text { Ar/N }\end{array}$ & 0.424 & 2.1328 \\
\hline $\begin{array}{c}\text { P11 CrAIN/CrAl } \\
40 / 60 \% \text { Ar/N }\end{array}$ & 0.265 & 1.7988 \\
\hline
\end{tabular}

Microscopía electrónica de barrido y composición química por espectrografía dispersiva de energía de rayos-X (EDS). 

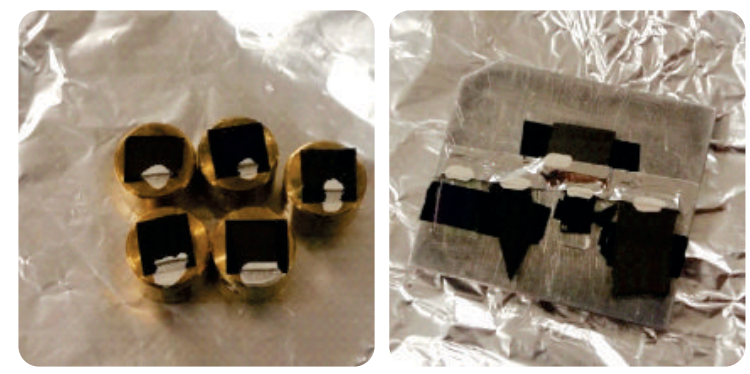

Figura 6. Muestras para realizar el análisis en el SEM (Izquierda) y EDS (Derecha)

Para realizar el análisis de la morfología de la superficie de las películas se usó un SEM (Microscopio electrónico de Barrido), se cortaron los sustratos en secciones de aproximadamente $5 \times 5 \mathrm{~mm}$. Para analizar el contenido químico se usó un equipo EDS (Espectroscopia de Energia Dispersiva), en este caso, se cortaron los sustratos en secciones de aproximadamente $1 \times 1 \mathrm{~cm}$, sujetándose al portamuestras por medio de cinta de grafito. En ambos casos, las superficies de las muestras son conectadas por medio de una cinta de aluminio y fijadas con pinturas de plata, además de un tratamiento térmico de un minuto con aire caliente y un minuto con aire frio.

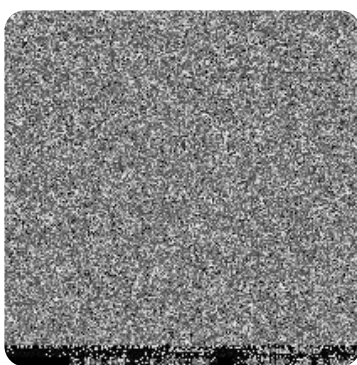

a)

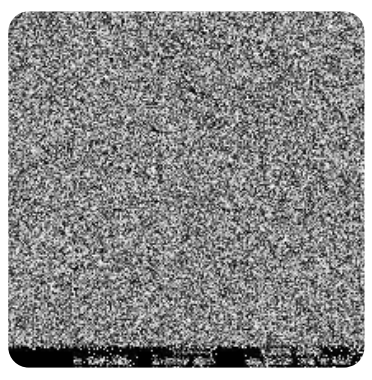

b)
Figura 7. Micrografías SEM de la superficie de dos películas analizadas.

Para realizar el análisis de la morfología de la superficie de las películas se usó un SEM (Microscopio electrónico de Barrido), se cortaron los sustratos en secciones de aproximadamente $5 \times 5 \mathrm{~mm}$. Para analizar el contenido químico se usó un equipo EDS (Espectroscopia de Energía Dispersiva), en este caso, se cortaron los sustratos en secciones de aproximadamente $1 \times 1 \mathrm{~cm}$, sujetándose al portamuestras por medio de cinta de grafito. En ambos casos, las superficies de las muestras son conectadas por medio de una cinta de aluminio y fijadas con pinturas de plata, además de un tratamiento térmico de un minuto con aire caliente y un minuto con aire frio.

\section{Tabla 3}

Resultados de porcentaje atómico de la técnica EDS

\begin{tabular}{|c|c|c|c|c|c|}
\hline $\begin{array}{c}\text { Muestral } \\
\text { Elemento }\end{array}$ & C & N & O & Al & \\
\hline $\begin{array}{c}\text { P3 CrAIN } \\
80 / 20 \% \text { Ar/N }\end{array}$ & 0 & $\begin{array}{r}33.206 \\
9953\end{array}$ & $\begin{array}{r}18.948 \\
0733\end{array}$ & $\begin{array}{r}17.488 \\
5444\end{array}$ & $\begin{array}{r}30.356 \\
387\end{array}$ \\
\hline $\begin{array}{c}\text { P10 CrAl/CrAIN } \\
40 / 60 \% \text { Ar/N }\end{array}$ & 0 & $\begin{array}{r}36.304 \\
9173\end{array}$ & $\begin{array}{r}23.338 \\
9865\end{array}$ & $\begin{array}{r}14.544 \\
0961\end{array}$ & 25.812 \\
\hline
\end{tabular}

Estos resultados también muestran que hay más presencia de cromo que de aluminio en las películas. Esto puede significar que el material no se formó o que no toda la película es del material deseado.

\section{CONCLUSIONES}

La técnica de magnetrón sputtering es un método eficiente para el depósito de películas delgadas en sustratos, porque se tiene el control de las condiciones de crecimiento para obtener recubrimientos de un material deseado, con un espesor fijo y con una fase cristalina deseada. En nuestro caso, fue posible depositar CrAIN sobre sustrato de vidrio para su análisis y caracterización y un sistema multicapas de CrAl/CrAIN, para obtener sus valores cuantitativos de sus principales propiedades. Los resultados de las caracterizaciones muestran que el método de síntesis CrAIN es correcto, sin embargo falta explorar en detalle algunas propiedades adicionales, tales como resistencia a la corrosión, dureza, desgaste que serán realizadas para completar este estudio. Además, debido a las condiciones de crecimiento, las películas recién depositadas fueron susceptibles a reacciones con el oxígeno del ambiente llegando a formar delgadas capas de óxido en la superficie de las mismas. 


\section{REFERENCIAS BIBLIOGRÁFICAS}

Beliardouh, N., Bouzid, K., Nouveau, C., Tlili, B., \& Walock, M. (2015). Tribological and electrochemical performances of $\mathrm{Cr} / \mathrm{CrN}$ and $\mathrm{Cr} / \mathrm{CrN} / \mathrm{CrAIN}$ multilayer coatings deposited by RF magnetron sputtering. Tribology International, 82(Part B), 443-452.

Bin, T., Xiaodong, Z., Naisai, H., \& Jiawen, $H$. (2000). Study on the structure and tribologicaal properties of $\mathrm{CrN}$ coatings by IBED. Surface and Coatings Technology, 131(1-3), 391-394.

Gonzáles Hernández, J., \& Espinoza Beltran, F. (2003). Materiales Superduros. Ciencia, Revista de la Academia Mexicana de Ciencias, 54(1), 10-17.

Gonzales, R., Cadenas, M., Fernandez, R., Cortizo, J., \& Rodriguez, E. (2007). Wear behaviour of flame sprayed NiCrBSi coating remelted by flame or by laser. Wear, 262, 301-307.

Holmberg, K., \& Mathews, A. (1994). Coatings tribology: a concept, critical aspects and future directions. Thin Solid Films, 253(1-2), 173-178.

Ipaz, L., Aperador, W., Caicedo, J., Esteve, J., \& Zambrano, G. (2012). A Practical Application of X-Ray Spectroscopy in Ti-Al-N and Cr-Al-N Thin Films. In S. K. Sharma (Ed.), X-Ray Spectroscopy (pp. 21-38): In Tech.

Li, P., Chen, L., Wang, S., Yang, B., \& Wu, M. (2013). Microstructure, mechanical and thermal properties of TiAIN/CrAIN multilayer coatings. International journal of Refractory Metals and Hard Materials, 40, 51-57.

Nouveau, C., Tlili, B., Aknouche, H.,

Benlatreche, Y., \& Patel, B. (2012). Comparison of CrAIN layers obtained with one (CrAl) or two targets $(\mathrm{Cr}$ and $\mathrm{Al})$ by magnetron sputtering. Thin Solid Films, $520(7), 2932-2937$.

Shigesato, Y., \& Paine, D. (1994). A microstructural study of low resistivity tindoped indium oxide prepared by d. c. magnetron sputtering. Thin Solid Films, 238(1), 44-50.

Velasco, F., Gordo, E., Isabel, R., RuizNavas, E., Bautista, A., \& Torralba, J. (2001). Mechanical and wear behaviour of highspeed steels reinforced with TiCN particles. International Journal of Metals and Hard Materials, 19(4-6), 319-323.

Xu, S., Long, J., Sim, L., Hoong, C., \& Ostrikov, K. (2005). RF Plasma Sputtering 53 Deposition of Hydroxyapatite Bioceramics: Synthesis, performance, and Biocompatibility. Plasma Processes and Polymers, 2(5), 373-390.

Xu, X., Wu, H., Zhang, C., \& Jin, Z. (2001). Morphological properties of AIN piezoelectric thin films deposited by $D C$ reactive magnetron sputtering. Thin Solid Films, 388(1-2), 62-67. 